\title{
Experimental Verification of Vuks Equation Using Hollow Prism Refractometer
}

\author{
Anita Kanwar ${ }^{1}$ and Priya S. Yadav ${ }^{2}$ \\ ${ }^{1}$ Department of Physics, VES College of Arts, Science and Commerce, Sindhi Society, Chembur, \\ Maharashtra, Mumbai 400071, India \\ ${ }^{2}$ Department of Physics, Atharva Engineering College, Malad West, Maharashtra, Mumbai 400095, India
}

Correspondence should be addressed to Anita Kanwar, anita_s_kanwar@yahoo.com

Received 31 July 2012; Revised 2 November 2012; Accepted 2 November 2012

Academic Editor: Boris A. Malomed

Copyright (๑) 2012 A. Kanwar and P. S. Yadav. This is an open access article distributed under the Creative Commons Attribution License, which permits unrestricted use, distribution, and reproduction in any medium, provided the original work is properly cited.

\begin{abstract}
The refractive indices of the cholesteric liquid crystal solution were measured using multiwavelength (visible range) refractometer for three different wavelengths. Measurements were made at different temperatures for various concentrations of the solution, mixing CLC in a soluble solvent. Vuks equation describes the wavelength and temperature dependence of refractive indices of anisotropic crystalline materials. We have used a simplified version of Vuks equation relating only to macroscopic indices and verified its validity for five-different-concentration solution at various temperatures. The result is also used to obtain molecular polarizabilities and temperature dependent material constants of our sample.
\end{abstract}

\section{Introduction}

The importance of liquid crystals lies in their thermal, electrical, and optical properties [1-3]. After understanding these properties one can hope to exploit the full range of possible device and materials applications. In many applications the knowledge of optical anisotropy $[4,5]$ and refractive indices of liquid crystals, and their temperature dependence is desirable [6]. Temperature-induced refractive index change is used in many liquid-crystal (LC) devices to modulate light [7]. Since LC shows optical anisotropy and is birefringent [8] in nature, its refractive index is quite different from that of an isotropic liquid. There are various methods used for the determination of refractive index of liquid crystals $[9,10]$.

Vuks [11] proposed a semiempirical model which is analogous to the classical Clausius-Mossotti equation for correlating the microscopic molecular polarizabilities to the macroscopic refractive indices of some crystalline materials. The Vuks paper is cited and used by many researchers to study properties of liquid crystals [12,13]. Vuks made a bold assumption that the internal field in a liquid crystal is the same in all directions and gave a semi-empirical equation correlating the refractive indices with the molecular polarizabilities for anisotropic materials [9]:

$$
\frac{n_{e, o}^{2}-1}{\left\langle n^{2}\right\rangle-2}=\frac{4 \pi}{3} N \alpha_{e, o},
$$

where $n_{e}$ and $n_{o}$ are the refractive indices for the extraordinary and ordinary ray, respectively, $\alpha_{e, o}$ are the corresponding molecular polarizabilities, $N$ is the number of molecules per unit volume, and $\left\langle n^{2}\right\rangle$ is given by

$$
\left\langle n^{2}\right\rangle=\frac{n_{e}^{2}+2 n_{o}^{2}}{3} .
$$

$\mathrm{Li}$ and $\mathrm{Wu}[14]$ modified this equation and showed that the validity of Vuks equations can be easily examined by measuring the temperature and wavelength-dependent refractive indices of liquid crystals. The modified equation (detailed derivation in [14]) given by them is

$$
\left\langle n^{2}\right\rangle+2 \approx \sqrt{10}\langle n\rangle-0.5,
$$

where average refractive index is

$$
\langle n\rangle=\frac{n_{e}+2 n_{0}}{3} .
$$


TABLE 1: Measured refractive indices $\left(n_{\mathrm{e}}\right.$ and $\left.n_{\mathrm{o}}\right)$ of 1-molar solution and validity of Vuks equation.

\begin{tabular}{|c|c|c|c|c|c|c|}
\hline Temp. (K) & $n_{\mathrm{e}}$ & $n_{\mathrm{o}}$ & $\langle n\rangle$ & $\left\langle n^{2}\right\rangle$ & LHS of $\left\langle n^{2}\right\rangle+2$ & RHS of $\sqrt{10}\langle n\rangle-0.5$ \\
\hline & \multicolumn{6}{|c|}{ Wavelength (404 nm) } \\
\hline 343 & 1.5156 & 1.4726 & 1.4869 & 2.2114 & 4.2114 & 4.2016 \\
\hline 333 & 1.5151 & 1.4767 & 1.4895 & 2.2189 & 4.2189 & 4.2098 \\
\hline 323 & 1.5186 & 1.4861 & 1.4969 & 2.2410 & 4.2410 & 4.2332 \\
\hline 313 & 1.5188 & 1.4916 & 1.5007 & 2.2522 & 4.2522 & 4.2452 \\
\hline \multirow[t]{2}{*}{303} & 1.5188 & 1.5027 & 1.5081 & 2.2743 & 4.2743 & 4.2686 \\
\hline & \multicolumn{6}{|c|}{ Wavelength (546 nm) } \\
\hline 343 & 1.5037 & 1.4577 & 1.473 & 2.1703 & 4.1703 & 4.1576 \\
\hline 333 & 1.5061 & 1.4705 & 1.4824 & 2.1977 & 4.1977 & 4.1873 \\
\hline 323 & 1.5044 & 1.4714 & 1.4824 & 2.1978 & 4.1978 & 4.1873 \\
\hline 313 & 1.5033 & 1.4775 & 1.4861 & 2.2086 & 4.2086 & 4.1990 \\
\hline \multirow[t]{2}{*}{303} & 1.5013 & 1.4853 & 1.4906 & 2.2220 & 4.2220 & 4.2133 \\
\hline & \multicolumn{6}{|c|}{ Wavelength $(578 \mathrm{~nm})$} \\
\hline 343 & 1.501 & 1.4547 & 1.4701 & 2.1618 & 4.1618 & 4.1485 \\
\hline 333 & 1.5046 & 1.4632 & 1.477 & 2.1819 & 4.1819 & 4.1703 \\
\hline 323 & 1.5025 & 1.4684 & 1.4798 & 2.1900 & 4.1900 & 4.1791 \\
\hline 313 & 1.5018 & 1.4735 & 1.4829 & 2.1993 & 4.1993 & 4.1889 \\
\hline 303 & 1.5084 & 1.4834 & 1.4917 & 2.2254 & 4.2254 & 4.2168 \\
\hline
\end{tabular}

TABLE 2: Measured refractive indices $\left(n_{\mathrm{e}}\right.$ and $\left.n_{\mathrm{o}}\right)$ of 2-molar solution and validity of Vuks equation.

\begin{tabular}{lcccccc}
\hline Temp. (K) & $n_{\mathrm{e}}$ & $n_{\mathrm{o}}$ & $\langle n\rangle$ & $n^{2}$ & LHS of $\left\langle n^{2}\right\rangle+2$ & RHS of $\sqrt{10}\langle n\rangle-0.5$ \\
\hline 343 & \multicolumn{5}{c}{ Wavelength $(404 \mathrm{~nm})$} \\
333 & 1.5137 & 1.4715 & 1.4856 & 2.2073 & 4.2073 & 4.1975 \\
323 & 1.512 & 1.4786 & 1.4897 & 2.2196 & 4.2196 & 4.2104 \\
313 & 1.5132 & 1.4873 & 1.4959 & 2.2380 & 4.2380 & 4.2300 \\
303 & 1.5134 & 1.4893 & 1.4973 & 2.2421 & 4.2512 & 4.2345 \\
\hline & 1.5131 & 1.494 & 1.5004 & 2.2512 & 4.2443 \\
\hline 343 & & & Wavelength $(546 \mathrm{~nm})$ & 4.1706 \\
333 & 1.5059 & 1.4627 & 1.4771 & 2.1822 & 4.2011 & 4.1911 \\
323 & 1.5056 & 1.4725 & 1.4836 & 2.2011 & 4.1867 & 4.1756 \\
313 & 1.5008 & 1.4676 & 1.4787 & 2.1867 & 4.2119 & 4.2025 \\
303 & 1.5085 & 1.4766 & 1.4872 & 2.2121 & 4.2025 \\
\hline 343 & 1.5067 & 1.4774 & 1.4872 & 2.2119 & 4.1787 & 4.1668 \\
333 & & & & Wavelength $(578 \mathrm{~nm})$ & 4.1785 \\
323 & 1.5045 & 1.4616 & 1.4759 & 2.1787 & 4.1789 & 4.1671 \\
313 & 1.5017 & 1.4686 & 1.4796 & 2.1896 & 4.2022 \\
303 & 1.4969 & 1.4656 & 1.476 & 2.1789 & 4.2053 & 4.1921 \\
\hline
\end{tabular}

The importance of modified Vuks equations (3) is that the sophisticated microscopic Vuks equation can now be validated by two simple macroscopic parameters: $\left\langle n^{2}\right\rangle$ and $\langle n\rangle$. These two parameters can easily be obtained by measuring the individual refractive indices $\left(n_{e}\right.$ and $\left.n_{o}\right)$ of the liquid crystal. In this paper we tried to verify this equation by finding out refractive indices of Cholesteric LC solution of five different concentrations prepared by us with varying its temperature.

\section{Experimental Method}

The most commonly used method to measure refractive indices is using an Abbe refractometer [15]. We have used hollow prism multiwavelength refractometer to measure refractive indices $[16,17]$ of a Cholesteric LC solution $\left(n_{e}\right.$ and $n_{o}$ ) and observed that these indices vary with both the temperature and the concentration. The solutions of five different molar concentrations were prepared using cholesteryl 
TABLE 3: Measured refractive indices $\left(n_{\mathrm{e}}\right.$ and $\left.n_{\mathrm{o}}\right)$ of 3-molar solution and validity of Vuks equation.

\begin{tabular}{lcccccc}
\hline Temp. (K) & $n_{\mathrm{e}}$ & $n_{\mathrm{o}}$ & $\langle n\rangle$ & $\left\langle n^{2}\right\rangle$ & LHS of $\left\langle n^{2}\right\rangle+2$ & RHS of $\sqrt{10}\langle n\rangle-0.5$ \\
\hline 343 & & & Wavelength $(404 \mathrm{~nm})$ & 4.2300 \\
333 & 1.5222 & 1.4828 & 1.4959 & 2.2382 & 4.2382 & 4.2487 \\
323 & 1.5187 & 1.4934 & 1.5018 & 2.2556 & 4.2546 & 4.2477 \\
313 & 1.5165 & 1.494 & 1.5015 & 2.2546 & 4.2494 & 4.2424 \\
303 & 1.5145 & 1.4924 & 1.4998 & 2.2494 & 4.2618 & 4.2553 \\
\hline & 1.5157 & 1.498 & 1.5039 & 2.2618 & 4.1892 \\
343 & & & Wavelength $(546 \mathrm{~nm})$ & 4.1998 & 4.1965 \\
333 & 1.5164 & 1.4663 & 1.483 & 2.1998 & 4.2065 & 4.2022 \\
323 & 1.5094 & 1.4733 & 1.4853 & 2.2065 & 4.1891 & 4.1782 \\
313 & 1.5074 & 1.4769 & 1.4871 & 2.2116 & 4.2163 & 4.2073 \\
303 & 1.4993 & 1.4696 & 1.4795 & 2.1891 & \\
\hline & 1.5074 & 1.4793 & 1.4887 & 2.2163 & 4.1785 & 4.1668 \\
343 & & & & Wavelength $(578 \mathrm{~nm})$ & \\
333 & 1.5012 & 1.4632 & 1.4759 & 2.1785 & 4.2054 & 4.1706 \\
323 & 1.5002 & 1.4655 & 1.4771 & 2.1820 & 4.1630 & 4.1500 \\
313 & 1.5069 & 1.474 & 1.485 & 2.2054 & 2.1630 & 4.2130 \\
303 & 1.4905 & 1.4607 & 1.4706 & 1.4905 & 2.2218 & \\
\hline
\end{tabular}

TABLE 4: Measured refractive indices $\left(n_{\mathrm{e}}\right.$ and $n_{\mathrm{o}}$ ) of 4-molar solution and validity of Vuks equation.

\begin{tabular}{|c|c|c|c|c|c|c|}
\hline Temp. (K) & $n_{\mathrm{e}}$ & $n_{\mathrm{o}}$ & $\langle n\rangle$ & $\left\langle n^{2}\right\rangle$ & LHS of $\left\langle n^{2}\right\rangle+2$ & RHS of $\sqrt{10}\langle n\rangle-0.5$ \\
\hline & \multicolumn{6}{|c|}{ Wavelength (404 nm) } \\
\hline 343 & 1.5189 & 1.477 & 1.491 & 2.2234 & 4.2234 & 4.2145 \\
\hline 333 & 1.5143 & 1.4751 & 1.4882 & 2.2150 & 4.2150 & 4.2057 \\
\hline 323 & 1.5135 & 1.4748 & 1.4877 & 2.2136 & 4.2136 & 4.2041 \\
\hline 313 & 1.5036 & 1.4751 & 1.4846 & 2.2042 & 4.2042 & 4.1943 \\
\hline \multirow[t]{2}{*}{303} & 1.5025 & 1.4757 & 1.4846 & 2.2043 & 4.2043 & 4.1943 \\
\hline & \multicolumn{6}{|c|}{ Wavelength $(546 \mathrm{~nm})$} \\
\hline 343 & 1.5128 & 1.4667 & 1.4821 & 2.1970 & 4.1970 & 4.1864 \\
\hline 333 & 1.499 & 1.4643 & 1.4759 & 2.1784 & 4.1784 & 4.1668 \\
\hline 323 & 1.4987 & 1.4641 & 1.4756 & 2.1778 & 4.1778 & 4.1658 \\
\hline 313 & 1.4987 & 1.4671 & 1.4776 & 2.1836 & 4.1836 & 4.1722 \\
\hline \multirow[t]{2}{*}{303} & 1.4969 & 1.4662 & 1.4764 & 2.1801 & 4.1801 & 4.1684 \\
\hline & \multicolumn{6}{|c|}{ Wavelength $(578 \mathrm{~nm})$} \\
\hline 343 & 1.4988 & 1.4567 & 1.4707 & 2.1635 & 4.1635 & 4.1504 \\
\hline 333 & 1.4932 & 1.4553 & 1.4679 & 2.1551 & 4.1551 & 4.1415 \\
\hline 323 & 1.4921 & 1.4542 & 1.4668 & 2.1519 & 4.1519 & 4.1380 \\
\hline 313 & 1.4892 & 1.4545 & 1.4661 & 2.1496 & 4.1496 & 4.1358 \\
\hline 303 & 1.4866 & 1.4538 & 1.4647 & 2.1457 & 4.1457 & 4.1314 \\
\hline
\end{tabular}

pelargonate and toluene as solvent. This refractometer is designed using a specially constructed hollow prism and optical spectrometer [18]. First the spectrometer is adjusted using optical leveling and Schuster's method. Angle of prism (A) is obtained. Then minimum deviation angles for three wavelengths: $404 \mathrm{~nm}, 546 \mathrm{~nm}$, and $578 \mathrm{~nm}$ of mercury source were measured. Refractive indices were calculated using the following formula:

$$
\mu=\frac{\sin ((A+\partial m) / 2)}{\sin (A / 2)} .
$$

A polarizer was used to identify ordinary and extraordinary spectrum. While to check the validity of the method initially refractive indices of few known liquids were obtained. A temperature variation was obtained using an indigenously designed heating coil and a digital thermometer. The same procedure was repeated for different concentrations of the mixture. For each concentration reading was taken with varying the temperature. The prism minimum deviation technique is commonly used for extremely accurate measurements $[19,20]$. Refractive index measurements correct up to 
TABLE 5: Measured refractive indices $\left(n_{\mathrm{e}}\right.$ and $\left.n_{\mathrm{o}}\right)$ of 5-molar solution and validity of Vuks equation.

\begin{tabular}{|c|c|c|c|c|c|c|}
\hline Temp. (K) & $n_{\mathrm{e}}$ & $n_{\mathrm{o}}$ & $\langle n\rangle$ & $\left\langle n^{2}\right\rangle$ & LHS of $\left\langle n^{2}\right\rangle+2$ & RHS of $\sqrt{10}\langle n\rangle-0.5$ \\
\hline & \multicolumn{6}{|c|}{ Wavelength (404 nm) } \\
\hline 343 & 1.518 & 1.4745 & 1.489 & 2.2175 & 4.2175 & 4.2082 \\
\hline 333 & 1.5154 & 1.4741 & 1.4879 & 2.2141 & 4.2141 & 4.2047 \\
\hline 323 & 1.5189 & 1.4839 & 1.4956 & 2.2370 & 4.2370 & 4.2291 \\
\hline 313 & 1.5128 & 1.491 & 1.4983 & 2.2449 & 4.2449 & 4.2376 \\
\hline \multirow[t]{2}{*}{303} & 1.5179 & 1.4998 & 1.5058 & 2.2676 & 4.2676 & 4.2613 \\
\hline & \multicolumn{6}{|c|}{ Wavelength $(546 \mathrm{~nm})$} \\
\hline 343 & 1.5104 & 1.4667 & 1.4813 & 2.1946 & 4.1946 & 4.1839 \\
\hline 333 & 1.5003 & 1.4617 & 1.4746 & 2.1747 & 4.1747 & 4.1627 \\
\hline 323 & 1.5118 & 1.475 & 1.4873 & 2.2123 & 4.2123 & 4.2028 \\
\hline 313 & 1.5058 & 1.4775 & 1.4869 & 2.2111 & 4.2111 & 4.2016 \\
\hline \multirow[t]{2}{*}{303} & 1.5013 & 1.4766 & 1.4848 & 2.2049 & 4.2049 & 4.1949 \\
\hline & \multicolumn{6}{|c|}{ Wavelength $(578 \mathrm{~nm})$} \\
\hline 343 & 1.5007 & 1.4567 & 1.4714 & 2.1654 & 4.1654 & 4.1526 \\
\hline 333 & 1.4965 & 1.4598 & 1.472 & 2.1672 & 4.1672 & 4.1545 \\
\hline 323 & 1.5075 & 1.4759 & 1.4864 & 2.2097 & 4.2097 & 4.2000 \\
\hline 313 & 1.4989 & 1.4679 & 1.4782 & 2.1854 & 4.1854 & 4.1741 \\
\hline 303 & 1.4977 & 1.4671 & 1.4773 & 2.1826 & 4.1826 & 4.1712 \\
\hline
\end{tabular}

TABLE 6: Variation of average refractive index with temperature for three wavelengths.

\begin{tabular}{lccc}
\hline$T(\mathrm{~K})$ & Average RI $(404 \mathrm{~nm})$ & Average RI $(546 \mathrm{~nm})$ & Average RI $(578 \mathrm{~nm})$ \\
\hline 343 & 1.48968 & 1.4793 & 1.4728 \\
333 & 1.49142 & 1.48036 & 1.47472 \\
323 & 1.49552 & 1.48222 & 1.4768 \\
313 & 1.49614 & 1.48346 & 1.47634 \\
303 & 1.50056 & 1.48554 & 1.48184 \\
\hline
\end{tabular}

TABle 7: Showing $A$ and $B$ values, for three wavelengths.

\begin{tabular}{lcc}
\hline Wavelength $(\mathrm{nm})$ & $A$ & $B\left(\mathrm{~K}^{-1}\right)$ \\
\hline 404 & 1.503 & $3.02 \times 10^{-4}$ \\
546 & 1.4865 & $1.65 \times 10^{-4}$ \\
578 & 1.4830 & $2.1 \times 10^{-4}$ \\
\hline
\end{tabular}

four to five decimal places are common using this technique with good control of the sample temperature.

\section{Observations}

Cholesteryl pelargonate in five different proportions was dissolved in the solvent to obtain different concentration solutions by properly stirring the solution at room temperature then heating it up to the isotropic temperature. Homogeneous solutions were heated, and refractive indices were obtained for every $10 \mathrm{~K}$ rise in the temperature using the multiwavelength refractometer. Using FPSS technique [21], we found that these solutions have nearly the same clearing temperature variations over the range of $1.5 \mathrm{~K}$, hence temperature in Kelvin and not reduced temperature is used in the calculations. We studied the variation of refractive index $\left(n_{e}\right.$ and $\left.n_{o}\right)$ with the temperature for various concentrations $[22,23]$. The results obtained are compiled in Tables 1, 2, 3, 4, and 5. The measurements were made for three different wavelengths in the visible range. The validity of the modified Vuks equation is checked for each sample.

Secondly we obtained average refractive index for each colour at different temperature (Table 6) using above data, and tried to correlate it with the equation given below according to which the average refractive index $\langle n\rangle$ decreases linearly as the temperature increases [24]. Figure 1 shows the variation of average refractive index for with temperature for three wavelengths. We also found the values of the constants $A$ and $B$ as per (6). These values are tabulated in Table 7 for three wavelengths used by us:

$$
\langle n\rangle=A-B T \text {. }
$$

We have also found the values of polarizabilities $\alpha_{e}$ and $\alpha_{o}$ for one molar solution using equations

$$
\begin{aligned}
& \alpha_{e}=\frac{3 M}{4 \pi \rho N_{A}} \cdot \frac{n_{e}^{2}-1}{\left\langle n^{2}\right\rangle+2} \\
& \alpha_{o}=\frac{3 M}{4 \pi \rho N_{A}} \cdot \frac{n_{o}^{2}-1}{\left\langle n^{2}\right\rangle+2},
\end{aligned}
$$


TABLE 8: Polarizabilities $\alpha_{\mathrm{e}}, \alpha_{\mathrm{o}}$, and $\langle\alpha\rangle$ in $\times 10^{-23} \mathrm{~cm}^{3}$ unit for one molar solution at various temperatures for three different wavelengths.

\begin{tabular}{lccccccrrr}
\hline \multirow{2}{*}{$T(\mathrm{~K})$} & \multicolumn{3}{c}{ Wavelength (404 nm) } & \multicolumn{3}{c}{ Wavelength (546 nm) } & \multicolumn{3}{c}{ Wavelength (578 nm) } \\
& $\alpha_{\mathrm{e}}$ & $\alpha_{\mathrm{o}}$ & $\langle\alpha\rangle$ & $\alpha_{\mathrm{e}}$ & $\alpha_{\mathrm{o}}$ & $\langle\alpha\rangle$ & $\alpha_{\mathrm{e}}$ & $\alpha_{\mathrm{o}}$ & \multicolumn{2}{c}{$\langle\alpha\rangle$} \\
\hline 343 & 6.7033 & 6.0392 & 6.2606 & 6.5818 & 5.8709 & 6.1078 & 6.5528 & 5.8372 \\
333 & 6.6835 & 6.0909 & 6.2884 & 6.5763 & 6.0269 & 6.2100 & 6.5777 & 5.9382 & 6.1513 \\
323 & 6.7032 & 6.2020 & 6.3691 & 6.5496 & 6.0405 & 6.2102 & 6.5321 & 6.0059 & 6.1813 \\
313 & 6.6887 & 6.2695 & 6.4092 & 6.5157 & 6.1180 & 6.2506 & 6.5068 & 6.0704 & 6.2158 \\
303 & 6.6541 & 6.4064 & 6.4889 & 6.4640 & 6.2177 & 6.2998 & 6.5689 & 6.1836 & 6.3121 \\
\hline
\end{tabular}

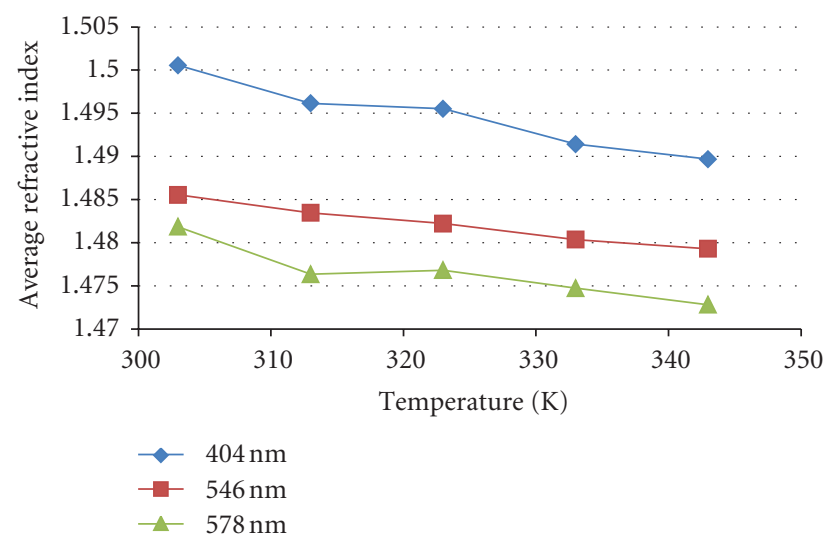

FIGURE 1: Shows the variation of average refractive index with temperature for three wavelengths.

where $\rho$ is the LC density, $M$ is the molecular weight (in our case it is 526.88), and $N_{A}$ is the Avogadro's number. The average value of $\alpha$ is obtained using the following equation:

$$
\langle\alpha\rangle=\frac{\left(\alpha_{e}+2 \alpha_{o}\right)}{3} .
$$

The values of $\alpha_{e}, \alpha_{o}$ and $\langle\propto\rangle$ are tabulated in Table 8 .

\section{Results and Discussion}

We have applied modified Vuks equation number (3) on five different solutions of Cholesteric liquid crystals and measured the extraordinary and the ordinary refractive indices using the hollow prism method. The experiments conducted by us not only validate the modified Vuks equation but also validate our method of finding refractive indices of liquid crystal solutions. There is an average error of nearly $0.2 \%$.

In the second part we are successful in obtaining values of the constants $A$ and $B$ for the equation which shows the variation of average refractive index with temperature. We are also able to show that average refractive index decreases with the increasing temperature linearly as specified by (6).

In the third part we have obtained values of average polarizability for three different wavelengths. The value of polarizability for Cholesteryl pelargonate predicted by chem.Spider data generated using the ACD/Labs, $\mathrm{ACD} /$ PhysChem Suite is $6.604 \times 10^{-23} \mathrm{~cm}^{3}$ at room temperature. This again is very close to the values calculated by us at various temperatures with the average error of 0.22 percent.
We have developed the hollow prism method to find refractive indices of liquid crystals. This method is validated by the simplified version of Vuks equation. Using this we are able to calculate birefringence, average refractive index, and molecular polarizabilities at various temperatures and concentrations.

\section{References}

[1] T. T. Alkeskjold, J. Lægsgaard, A. Bjarklev et al., "All-optical modulation in dye-doped nematic liquid crystal photonic bandgap fibers," Optics Express, vol. 12, no. 24, pp. 5857-5871, 2004.

[2] I. C. Khoo, "Nonlinear optics of liquid crystalline materials," Physics Reports, vol. 471, no. 5-6, pp. 221-267, 2009.

[3] J. Yan, L. Rao, M. Jiao, Y. Li, H. C. Cheng, and S. T. Wu, "Polymer-stabilized optically isotropic liquid crystals for nextgeneration display and photonics applications," Journal of Materials Chemistry, vol. 21, no. 22, pp. 7870-7877, 2011.

[4] W. H. de Jeu, "Physical properties of liquid crystalline materials in relation to their applications," Molecular Crystals and Liquid Crystals, vol. 63, no. 1, pp. 83-109, 1980.

[5] N. V. S. . Rao, V. G. K. M. Pisipati, P. V. Datta Prasad, and P. R. Alapati, "Order parameter and molecular polarizabilities in 60.4, 60.2 and 70.1," Molecular Crystals and Liquid Crystals, vol. 132, no. 1-2, pp. 1-21, 1986.

[6] B. Bahadur, Ed., Liquid Crystals: Applications and Uses, vol. 1, World Scientific, New Jersey, NJ, USA, 1990.

[7] N. V. Tabiryan, A. V. Sukhov, and B. Y. Zel'dovich, "Orientational optical nonlinearity of liquid crystals," Molecular Crystals and Liquid Crystals, vol. 136, no. 1, pp. 1-139, 1986.

[8] C. Motoc and G. Iacobescu, "Birefringence of azo-dye doped nematic liquid crystals," Journal of Optoelectronics and Advanced Materials, vol. 8, no. 1, pp. 295-298, 2006.

[9] A. K. Singh, R. Manohar, J. P. Shukla, and A. M. Biradar, "Refractive indices, order parameter and optical transmittance studies of a nematic liquid crystal mixture," Acta Physica Polonica A, vol. 110, no. 4, pp. 485-493, 2006.

[10] R. Manohar, A. Kumar Srivastava, Jyotishman, J. P. Shukla et al., "Dielectric, optical and thermodynamical properties of liquid crystal sample exhibiting SmA phase," International Journal of Physical Sciences, vol. 1, no. 3, pp. 147-153, 2006.

[11] M. F. Vuks, "Determination of the optical anisotropy of aromatic molecules from the double refraction of crystals," Optics and Spectroscopy, vol. 20, p. 361, 1966.

[12] M. Roushdy, "Properties and thermal phase behaviour of binary mixtures of homologues of unsymmetrically substituted phenyl benzoates," Egyptian Journal of Solids, vol. 29, no. 2, pp. 227-240, 2006.

[13] J. R. D. Pereira, A. M. Mansanares, A. J. Palangana, and M. L. Baesso, "Temperature dependence of the refractive index near 
the reentrant-isotropic-calamitic-nematic phase transition," Physical Review E, vol. 64, no. 1, Article ID 012701, 4 pages, 2001.

[14] J. Li and S.-T. Wu, "Self-consistency of Vuks equations for liquid-crystal refractive indices," Journal of Applied Physics, vol. 96, no. 11, pp. 6253-6258, 2004.

[15] J. Li, C.-H. Wen, S. Gauza et al., "Refractive indices of liquid crystals for display applications," Journal of Display Technology, vol. 1, no. 1, pp. 51-61, 2005.

[16] G. Sureshchandra, P. Prabhu, A. Singh et al., "Studies of nanoparticle doped liquid crystal mixtures," Molecular Crystals and Liquid Crystals, vol. 511, no. 1, pp. 75/[1545]-84/[1554], 2009.

[17] A. Kanwar and P. S. Yadav, "Temperature and concentration dependence of refractive index of cholesteric liquid crystal solution," Physical Chemistry: An Indian Journal, vol. 7, no. 1, pp. 36-42, 2012.

[18] S. Singh, "Refractive index measurement and its applications," Physica Scripta, vol. 65, no. 2, pp. 167-180, 2002.

[19] R. Gupta, J. H. Burnett, U. Griesmann, and M. Walhout, "Absolute refractive indices and thermal coefficients of fused silica and calcium fluoride near $193 \mathrm{~nm}$," Applied Optics, vol. 37, no. 25, pp. 5964-5968, 1998.

[20] M. R. Shenoy, S. Roy, and K. Thyagarajan, "Simple prism coupling technique to measure the refractive index of a liquid and its variation with temperature," Review of Scientific Instruments, vol. 61, no. 3, pp. 1010-1013, 1990.

[21] J. Gupta Sureshchandra, "New mesophase transitions in cholesteryl chloride using an optical method," Indian Journal of Pure and Applied Physics, vol. 37, no. 8, pp. 620-621, 1999.

[22] T. N. Soorya, S. Gupta, A. Kumar, S. Jain, V. P. Arora, and B. Bahadur, "Temperature dependent optical property studies of nematic mixtures," Indian Journal of Pure and Applied Physics, vol. 44, no. 7, pp. 524-531, 2006.

[23] J. H. Burnett, R. Guta, and U. Griesmann, "Absolute refractive indices and thermal coefficients of $\mathrm{CaF}_{2}, \mathrm{SrF}_{2}, \mathrm{BaF}_{2}$, and $\mathrm{LiF}$ Near 157 nm," Applied Optics, vol. 41, no. 13, pp. 2508-2513, 2002.

[24] J. Li, S. Gauza, and S. T. Wu, "Temperature effect on liquid crystal refractive indices," Journal of Applied Physics, vol. 96, no. 1, p. 19, 2004. 

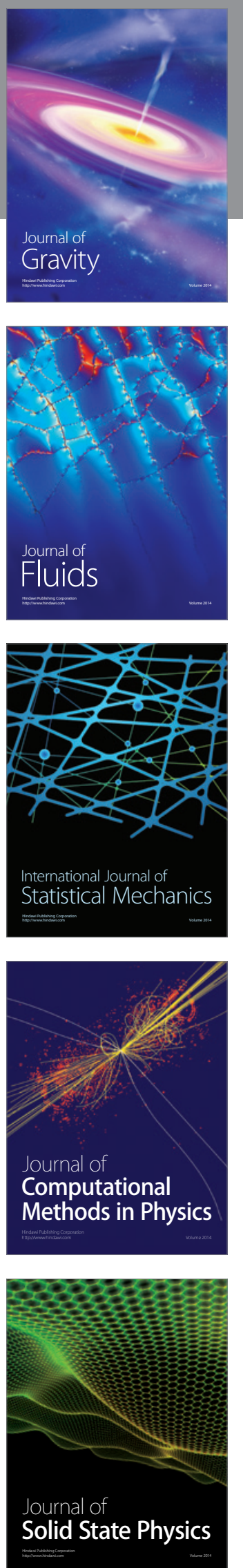

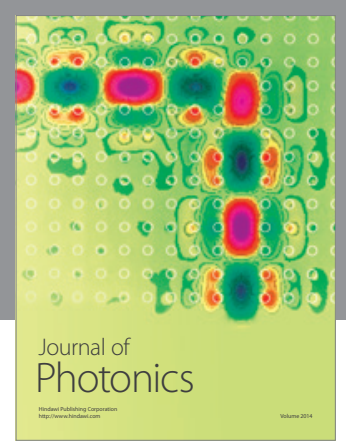

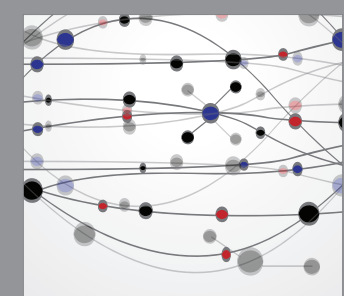

The Scientific World Journal
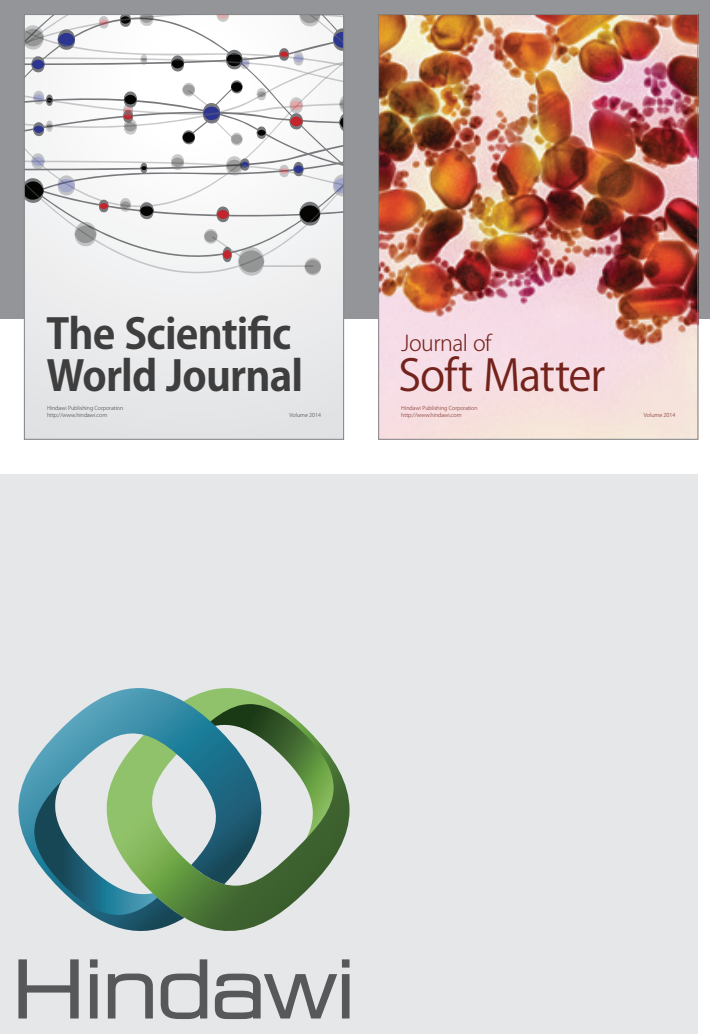

Submit your manuscripts at

http://www.hindawi.com
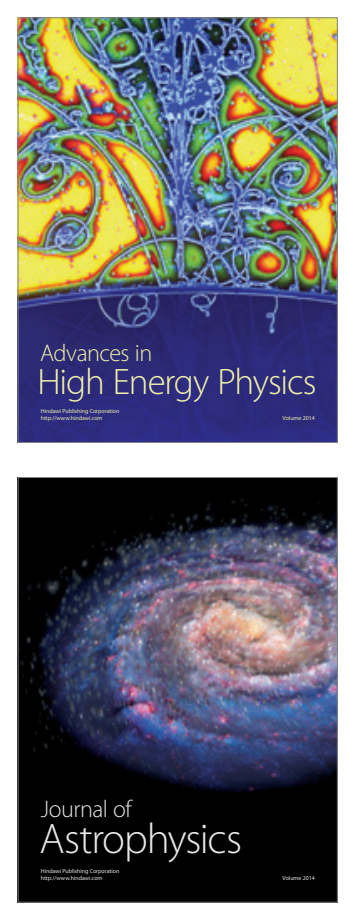
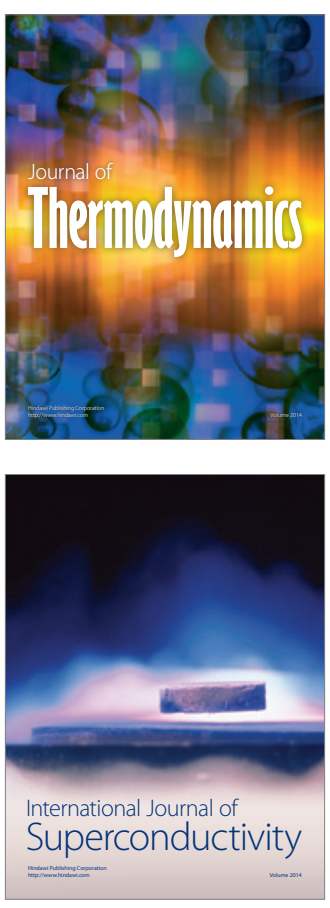
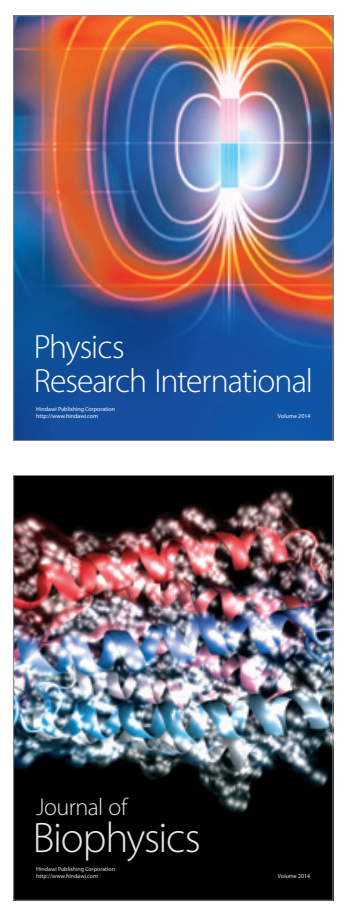
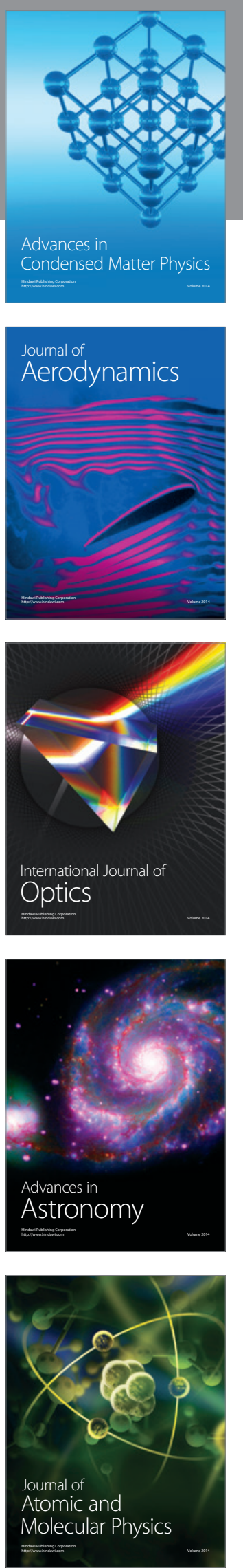